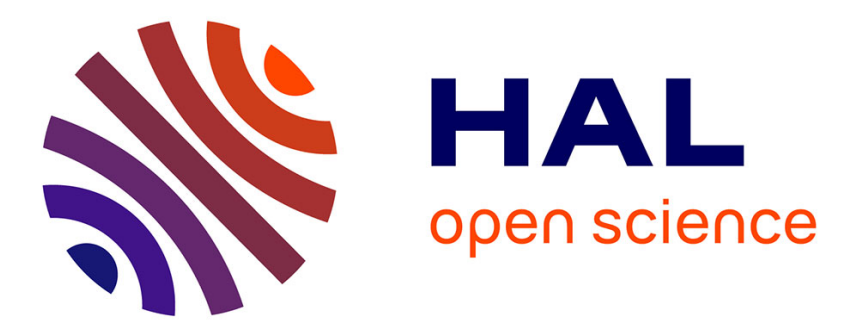

\title{
Overweight in mice, induced by perinatal programming, exacerbates doxorubicin and trastuzumab cardiotoxicity
}

Charles Guenancia, Olivier Hachet, Mona Aboutabl, Na Li, Eve Rigal, Yves Cottin, Luc Rochette, Catherine Vergely

\section{- To cite this version:}

Charles Guenancia, Olivier Hachet, Mona Aboutabl, Na Li, Eve Rigal, et al.. Overweight in mice, induced by perinatal programming, exacerbates doxorubicin and trastuzumab cardiotoxicity. Cancer Chemotherapy and Pharmacology, 2016, 77 (4), pp.777 - 785. 10.1007/s00280-016-2995-9 . hal03433403

\section{HAL Id: hal-03433403 \\ https://u-bourgogne.hal.science/hal-03433403}

Submitted on 18 Nov 2021

HAL is a multi-disciplinary open access archive for the deposit and dissemination of scientific research documents, whether they are published or not. The documents may come from teaching and research institutions in France or abroad, or from public or private research centers.
L'archive ouverte pluridisciplinaire HAL, est destinée au dépôt et à la diffusion de documents scientifiques de niveau recherche, publiés ou non, émanant des établissements d'enseignement et de recherche français ou étrangers, des laboratoires publics ou privés. 


\section{Overweight in mice, induced by perinatal programming, exacerbates doxorubicin and trastuzumab cardiotoxicity}

Charles Guenancia ${ }^{\text {a, b }}$, Olivier Hachet ${ }^{\text {a, b }}$, Mona Aboutabl ${ }^{\text {a, c }}, \mathrm{Na} \mathrm{Li}^{\text {a }}$, Eve Rigal ${ }^{\text {a }}$, Yves Cottin ${ }^{\text {a, b }}$, Luc Rochette ${ }^{a}$ and Catherine Vergely ${ }^{a}$

${ }^{a}$ Laboratoire de Physiopathologie et Pharmacologie Cardio-Métaboliques (LPPCM), Inserm UMR866, University of Bourgogne-Franche-Comte, Faculties of Health Sciences, Dijon, France

${ }^{b}$ Cardiology Department, University Hospital, Dijon, France

${ }^{c}$ Medicinal and Pharmaceutical Chemistry Department (Pharmacology group), Pharmaceutical and Drug Industries Research Division, National Research Centre (ID: 60014618), 33 El Bohouth St., Dokki, Giza, Egypt P.O. 12622

Corresponding author: Pr. Catherine VERGELY, LPPCM, Inserm UMR866, 7 Bd Jeanne d'Arc, 21000 Dijon, France

Telephone: (+33) 380393 460, Fax: (+33) 380393293

Email: cvergely@u-bourgogne.fr

Funding: This work was supported by grants from the French Ministry of Research, the Institut National de la Santé et de la Recherche Médicale (INSERM), the Société Française de Cardiologie (SFC), the Regional Council of Burgundy, the Association de Cardiologie de Bourgogne (ACB) and the French Government through a fellowship granted by the French Embassy in Egypt (Institut Français d'Egypte). 


\section{ABSTRACT}

Purpose: Trastuzumab (TRZ) is believed to potentiate doxorubicin (DOX) cardiotoxicity, resulting in left ventricular dysfunction. There is some evidence that overweight could influence anticancer drug-induced cardiotoxicity, though no study has evaluated the impact of moderate overweight, induced by postnatal nutritional programming, on the cardiotoxic effects of DOX alone or in combination with TRZ.

Methods: Immediately after birth, litters of C57BL/6 mice were either maintained at 9 pups (normal litter, NL), or reduced to 3 (small litter, SL) in order to induce programming of $\sim 15 \%$ overweight through postnatal overfeeding. At 4 months, NL and SL mice received a single intraperitoneal injection of either saline, DOX (6 mg/kg), or TRZ $(10 \mathrm{mg} / \mathrm{kg})$ or both (DOX-TRZ). Transthoracic echocardiography was performed 24 hours before as well as 10 and 20 days after treatments.

Results: Twenty days after DOX administration, systolic dysfunction was observed only in the overweight SL group, while NL mice group had a normal left ventricular ejection fraction (LVEF). However, in the NL group, functional impairment appeared when TRZ was co-administered. Forty-eight hours after drug administration, gene expression of natriuretic peptides (ANP, BNP) appeared to be potentiated in DOX-TRZ mice of both the NL and SL group, whereas the expression of $\beta-\mathrm{MHC}$ increased significantly in overweight-SL mice only.

Conclusions: In an acute model of DOX cardiotoxicity, moderately overweight adult mice were more sensitive to cardiac systolic impairment. Moreover, our results confirm the potentiating action of TRZ on DOX-induced cardiotoxicity in lean mice.

Keywords: Doxorubicin, trastuzumab, cardiotoxicity, overweight, postnatal programming 


\section{Introduction}

Doxorubicin (DOX, Adriamycin ${ }^{\circledR}$ ) is an anthracycline with a broad spectrum of activity in oncological practice [1]; however, its use is limited by its dose-dependent cardiotoxicity, which may result in both acute and chronic cardiotoxic effects [2,3]. Following the administration of anthracyclines in the adjuvant settings of breast cancer, trastuzumab (TRZ), a humanized monoclonal antibody against the human epidermal growth factor receptor 2 (HER 2) protein [4], is effective in patients that overexpress this receptor. The association of TRZ and chemotherapy provides a clinical benefit in terms of recurrence-free survival and overall survival compared with chemotherapy alone [5-8]. Nonetheless, while preclinical studies did not reveal cardiotoxicity [9], later clinical studies showed that treatment with TRZ led to an unexpected incidence of cardiac side effects [10]. The most frequent effect was reduced systolic ventricular function, which was asymptomatic or associated with heart failure [11].

In fact, clinical trials have shown a greater incidence of cardiac dysfunction when anthracycline chemotherapy was associated with TRZ than with chemotherapy alone [10] and TRZ is now believed to potentiate the cardiotoxic effects of anthracyclines [6,7,12]. In in vivo murine models that combined DOX with TRZ, a synergetic interaction leading to deterioration in cardiac function [13] and to an increase in cardiomyocyte apoptosis was reported [14,15]. In addition, in vitro in cardiomyocytes, the blockage of HER2 was accompanied by apoptotic processes and by an increase in the level of oxidative stress possibly through mitochondrial and reactive oxygen speciesdependent pathways $[16,17]$.

Several factors may increase the incidence of cancer in overweight and obese individuals [18]. Although it has been reported for many years that patients with metabolic syndrome have a higher risk of cardiovascular disease [19], their susceptibility to chemotherapy-induced cardiac disease has only recently been documented. In fact, few studies have shown that obese patients [20] or rats fed with a high-lipid diet [21] are more sensitive to the cardiotoxic effects of anthracyclines. Moreover, 
obesity has recently been associated with a poor outcome in breast cancer patients treated with DOX-based chemotherapy [22,23], a finding that could in part be related to greater sensitivity to the side-effects of anticancer agents. Therefore, it is important to explore more deeply the influence of overweight and obesity as aggravating factors in the cardiotoxic effects of anticancer drugs, particularly when several types of chemotherapy are combined.

We recently demonstrated that overfeeding during the immediate postnatal period in mice induced perinatal programming of cardio-metabolic risk. Indeed, postnatal overfeeding not only induced permanent moderate overweight, but also, during adulthood, increased blood pressure, glucose intolerance and insulin-resistance [24]. This rodent model, which presents several features of mild metabolic syndrome, is also characterized by greater susceptibility to myocardial ischemiareperfusion injury $[25,26]$. Therefore, this model is potentially relevant to study the impact of overweight on heart sensitization to the cardiotoxic effects of anticancer drugs, and particularly the association of anthracyclines with TRZ.

Thus, the aim of our study was to evaluate in mice the role of overweight in the cardiac impact of acute DOX treatment associated or not with TRZ. 


\section{Material and methods}

\subsection{Animals}

Adult female C57BL/6 mice (Charles River, L'Arbresle, France) were mated with male mice, then housed in individual cages and given water and standard chow ad libitum during pregnancy and lactation. On day-2 after birth, male pups were randomly distributed among the mothers and the litter size was adjusted to 9 pups (normal litter, NL group), or reduced to 3 pups in order to induce postnatal-overfeeding (small litter, SL group) as previously described [25]. The investigation was approved by the local ethics committee (Comité d'Ethique de l'Expérimentation Animale, Université- Bourgogne-Franche-Comté, Dijon, France, protocol agreement number: 00412.03). Throughout the procedure, care was taken to avoid suffering and to ensure animal welfare.

\subsection{Treatments}

At 4 months of age, considered as adulthood for mice, control groups originating from NL and overweight groups from SL were subjected to different types of treatment.

A preliminary study was conducted to determine the acute dose of DOX that could impair cardiac contractile function in both groups of mice. For this purpose, DOX (Sigma-Aldrich, St Louis, MO) was tested at 0,6 and $10 \mathrm{mg} / \mathrm{kg}$ (intra-peritoneal (IP) single injection) in $29 \mathrm{NL}$ and $28 \mathrm{SL}$ mice. These low-doses of DOX were chosen because of their mild impact on mortality, which is the case for doses up to $15 \mathrm{mg} / \mathrm{kg}$ [27]. All mice underwent transthoracic echocardiography and our results showed that in mice treated with $6 \mathrm{mg} / \mathrm{kg}$ DOX, the impairment of systolic function appeared only in overweight SL mice. At $10 \mathrm{mg} / \mathrm{kg}$, DOX induced systolic dysfunction at D10 and D20 in all groups. Therefore, in order to determine the possible deleterious interaction between DOX and TRZ, the dose of $6 \mathrm{mg} / \mathrm{kg}$ of DOX was chosen for the following experiments.

In order to assess the impact of overweight on the development of cardiotoxicity by DOX, TRZ or their association, 74 NL mice and 79 SL mice aged 4 months received at D1 a single IP injection of 
either saline solution $(\mathrm{NaCl} 0.9 \%)$, or DOX (6 mg/kg), or TRZ (10 mg/kg [14,27,28]) or DOX (6 $\mathrm{mg} / \mathrm{kg})+\mathrm{TRZ}(10 \mathrm{mg} / \mathrm{kg})$. Thus, 8 groups were constituted (Figure 1).

Body weight was measured at baseline, before each echocardiography and before sacrifice. Transthoracic echocardiography was performed 24 hours before (day 0, D0), ten (D10) and twenty (D20) days after the treatments. A subset of 6 mice per group was euthanized 48 hours after the injections (D3), in order to evaluate early circulating and cardiac modifications. At the end of the protocol (D20), all of the animals were euthanized after anaesthesia under pentobarbital (60 mg/kg, IP); blood and tissue were harvested, immediately frozen in liquid nitrogen and stored at $-80 \mathrm{C}$ until further analysis.

\subsection{Transthoracic echocardiographic imaging}

Transthoracic echocardiography was performed using the Vevo $770^{\mathrm{TM}}$ imaging system (VisualSonics Inc., Toronto, Canada), equipped with a $30 \mathrm{MHz}$ probe as described previously [29]. Briefly, mice were anesthetized with isoflurane and their body temperature was maintained at $37 \pm 0.5^{\circ} \mathrm{C}$. An ECG signal was also monitored. The ultrasound probe (RMV-707B) was placed on the mouse's chest. Two-dimensional images were recorded in the parasternal long- and short-axis views. Pulsed Doppler studies of LV diastolic function were performed in the apical 4-chamber view with the Doppler cursor oriented parallel to the long-axis plane of the left ventricle.

\subsection{Quantitative measurement of BNP and cTnI}

Plasma brain natriuretic peptide (BNP) and cardiac troponin I (cTnI) levels were determined with sandwich enzyme immunoassays (Uscn, Life science Inc) on the VICTOR3-V multilabel reader (Wallac-PerkinElmer Life Sciences, Courtaboeuf, France) according to the instructions of the manufacturer (Cloud-Clone Corp., Houston, USA). 


\subsection{Myocardial gene expression}

Total RNA was extracted from frozen heart samples and real-time quantitative polymerase chain reaction (RT-PCR) was performed as described before [25]. Cardiac mRNA levels of atrial natriuretic peptide (ANP), brain natriuretic peptide (BNP) and beta-myosin heavy chain ( $\beta$-MHC) were normalized against the housekeeping hypoxanthine-guanine phosphoribosyltransferase (HPRT) gene expression. The primers used for the amplification of mouse genes are provided in Table 1.

\subsection{Statistical analysis}

Continuous data were expressed as means \pm standard deviations (SD) and dichotomous data as numbers (percentages). For continuous variables, the normality of distribution was tested by the Shapiro-Wilk test. For body weight and echocardiographic data, values were compared using 2-way ANOVA for repeated measures followed by a Tukey post-hoc analysis. Because of the presence of two factors in treatment groups (SL vs NL and the treatment administered), all other continuous data were compared using 2-way ANOVA. This allowed the analysis of the effects of TRZ and DOX, and the potential interaction of these two factors. Post-hoc analyses were done with Tukey test if appropriate. 


\section{Results}

\section{1. $\quad$ Mice body and heart weight}

At D0, aged 4 months, SL mice were almost $14 \%$ heavier than NL mice (32.6 g vs. $28.8 \mathrm{~g}, \mathrm{p}<0.001)$ and this difference persisted throughout the duration of the experiment (Figure 2).

The administration of DOX, alone or in combination with TRZ, induced a transient decrease in body weight at D10 in the SL groups but not in the NL groups (Figure 2B and 2D).

In control groups, there was a significant reduction of the ratio of left ventricular weight to body weight (Table 2) at any time point in SL groups as compared to NL, due to the fact that body weight was increased, but that heart weight was not significantly modified. No changes were induced by DOXO or TRZ alone between DO and D10 and/or D20. However, a significant increase in the ratio was observed at D 20 in both the NL and SL groups, revealing myocardial hypertrophy.

\subsection{Cardiac function in vivo}

The echocardiographic measurements are sumarized in Table 3.

At D0, the left ventricular ejection fraction (LVEF) in NL groups was no different from that in SL groups (Table 3). LVEF remained unchanged over time in CONT saline-treated animals (Figure 3A). DOX did not modify the LV systolic function in the NL group (NL DOX) at D10 or D20 (Figure 3B). However, in the SL DOX group, there was a significant decrease in LVEF at D10 (49\% \pm 7 vs. $61 \% \pm 5)$, which persisted at D20 (47\% \pm 4$)$. TRZ alone did not alter LVEF in either the NL or SL groups (Figure 3C). The administration of a combined treatment with DOX and TRZ modified LVEF in both groups: at D10, there was a significant decrease in LVEF only in the SL DOX-TRZ (49\% \pm 4 vs $58 \% \pm 3)$ group but at D20 (48\% \pm 5$)$, the systolic function was similarly affected in both NL and SL groups. At D20, LVEF values in the NL DOX-TRZ group were significantly lower than those in the NL DOX group $(52 \% \pm 6$ vs $58 \% \pm 3$, p $<0.01)$ whereas there was no significant difference between the SL DOX-TRZ and SL DOX groups. 
In the DOX and DOX-TRZ groups, left ventricular diameter (LVEDD) in SL mice was higher than that in NL mice at D10 and D20 (p<0.005), suggesting more severe cardiac alteration.

In the present study, we did not observe the development of an abnormal diastolic function profile for mice treated with DOX and TRZ (E/A or E/E').

\subsection{Quantitative measurement of plasma cTnI and BNP}

Cardiac troponin I and BNP levels were measured in blood plasma collected by intra-cardiac puncture at D3 and D20. These results showed low or undetectable levels that were similar in all groups of mice (data not shown).

\subsection{Myocardial expression of genes involved in remodelling and heart failure}

The mRNA expression of BNP, ANP and $\beta$-MHC were measured in myocardial tissue harvested 48 hours after treatment (D3) or at the end of the experimental protocol (D20).

Our result showed early but not late expression of these genes. At D3, myocardial expression of ANP and BNP was significantly higher in NL and SL groups treated with the combination of DOX and TRZ than in the untreated groups (Figure 4A and 4B). The combined treatment induced significantly higher ANP gene expression than did DOX or TRZ alone (Figure 4B) in both SL and NL groups.

Conversely, an increase in $\beta$-MHC expression was only observed in overweight SL groups treated with the combination of DOX and TRZ, but not in lean NL groups nor in mice treated with DOX or TRZ alone (Figure 4C). 


\section{Discussion}

The main objective of our study was to investigate the role of moderate overweight in mice in the cardiac consequences of DOX treatment, in combination or not with TRZ. The results clearly confirmed, through the evaluation of cardiac function with in vivo echocardiography, that overweight mice are more prone to DOX-induced cardiotoxicity than their lean counterparts, as they show earlier impairment of LVEF and increased ventricular diameters. TRZ potentiated the cardiac effects of DOX, as shown by higher heart-to-body weight, a marker of myocardial hypertrophy, a higher myocardial expression of genes involved in heart failure (ANP, BNP), and increased impairment of left ventricular function in lean NL mice. To our knowledge, this is the first study to evaluate, in a mouse model of postnatal programming of "cardio-metabolic risk", the consequences of anticancer drugs, and to show that overweight individuals are more sensitive to anthracycline cardiotoxicity.

One of the major findings of our study is that moderately overweight SL adult mice present a greater likelihood of developing cardiomyopathy with systolic dysfunction for very low doses of DOX (6 mg/kg), whereas normal weight NL mice did not. For the higher $10 \mathrm{mg} / \mathrm{kg}$ dose of DOX, impairment of left ventricular function was observed in all groups at D10 and D20. In our study, in order to acquire multiple echocardiographic images over time, we intentionally used low doses of DOX that did not cause death, but were still able to induce acute cardiac toxicity. As a comparison with the clinical situation, in the management of acute leukaemia, for which intensive combination chemotherapy schedules are employed, the recommended dose of doxorubicin is $2.4 \mathrm{mg} / \mathrm{kg}$ of body weight (approximately corresponding to $75-90 \mathrm{mg} / \mathrm{m}^{2}$ ), to be administered divided over three consecutive days (one cycle).

The results presented here suggest that our murine model of moderate overweight, obtained by postnatal programming through over-nutrition during the suckling period, is highly sensitive to 
DOX-induced cardiotoxicity. To the best of our knowledge, no previous study has shown that moderate overweight could aggravate DOX-mediated cardiotoxicity. Our results could be linked to those of Mitra et al., who reported evidence of increased DOX-cardiotoxicity in a rodent model of obesity [21]. In their work, male rats were obese (30\% overweight) after 43 -days of a $40 \%$ high-fat diet and cardiotoxicity was evaluated after a single IP dose of DOX $(8 \mathrm{mg} / \mathrm{kg})$. The results showed that while only $10 \%$ of the normal diet rats died after DOX administration, $80 \%$ of the obese rats died, thus exhibiting the increased sensitivity of obese rats to DOX-induced toxicity. Our study, using both functional and molecular cardiac assessments, supports their hypothesis and emphasizes the major role of overweight in DOX cardiotoxicity.

The mechanisms by which obesity is able to promote DOX-induced cardiotoxicity must be specified with further studies. In a study from our laboratory, we reported that overweight 6-monthold rodents had higher cardiac susceptibility to ischemia-reperfusion injury, increased cardiac oxidative stress and metabolic alterations such as increased leptin and decreased adiponectin levels [26]. From a clinical point of view, obesity is an established risk factor for several cancers [30]. Unfortunately, the occurrence of cardiac events during anthracycline-based chemotherapy has almost never been recorded in trials. Thus, clinical data are required to determine whether obese patients are more prone than non-obese patients to the cardiotoxicity of DOX or TRZ treatments.

Our results showed that TRZ could potentiate the cardiotoxicity induced by DOX, especially in the context of lean NL mice. Indeed, this finding was supported both by increased and earlier deterioration of systolic function and by early elevation of markers of heart failure and remodelling (ANP, BNP and $\beta$-MHC) in hearts of mice treated with a combination of TRZ and DOX. Walker et al. [27] evaluated over 10 days mice receiving a combination of DOX (20 mg/kg) and TRZ (10 $\mathrm{mg} / \mathrm{kg}$ ). Although the mortality rate for DOX-treated mice was high (50\%) at day 5, the mortality rate was even worse for DOX+TRZ-treated mice (80\%). Jassal et al. [14], using an acute murine model of chemotherapy-induced cardiotoxicity, observed that the mortality rate was extremely high 
$(80 \%)$ in DOX+TRZ treated mice compared with that in mice with DOX treatment only. Mice treated with DOX or DOX+TRZ demonstrated progressive LV dilatation and reduced LVEF, compared with the group control, but systolic dysfunction appeared earlier in the group treated with DOX+TRZ.

The pharmacological target of TRZ in mice is still a matter of great debate. The potentiation of DOX-induced cardiomyopathy by TRZ could be explained by the impossibility of HER-2 survival pathways to overreact to the anthracycline-related stress in cardiomyocytes. Indeed, in mice, TRZinduced cardiotoxicity has been attributed to the blockade of HER-2 signalling in cardiomyocytes, which lead to the alteration of antiapoptotic signalling pathways [27] and to an increase in oxidative stress. Gordon et al. showed that blockade of HER-2 enables the accumulation of reactive oxygen species (ROS) within neonatal rats' cardiomyocytes through a mitochondrial pathway, leading to the development of cardiac dysfunction by stimulating cardiomyocyte apoptosis [16]. In addition, increased angiotensin II levels as a result of HER-2 inhibition lead to an increase in ROS production and the inhibition of neuregulin signalling [31].

\section{Conclusion}

Our model of postnatal programming suggests, for the first time, an association between overweight and DOX-induced cardiotoxicity, resulting in greater sensitivity to the cardiotoxic effects of DOX, with significant acute systolic function impairment assessed by echocardiography. Furthermore, our results confirmed that TRZ administration potentiates the acute cardiotoxicity induced by DOX, especially in lean mice treated with DOX. 
Acknowledgements: The authors thank Françoise Bechet for technical assistance and Mr.

Philip Bastable for English revision of the manuscript.

Conflict of interest: On behalf of all authors, the corresponding author states that there is no conflict of interest including financial and personal relationships with other people or organizations that could inappropriately influence this work. 


\section{References}

1. Pommier Y, Leo E, Zhang H, Marchand C (2010) DNA topoisomerases and their poisoning by anticancer and antibacterial drugs. Chem Biol 17 (5):421-433. doi:10.1016/j.chembiol.2010.04.012

2. Singal PK, Iliskovic N (1998) Doxorubicin-induced cardiomyopathy. N Engl J Med 339 (13):900-905. doi:10.1056/NEJM199809243391307

3. Keefe DL (2001) Anthracycline-induced cardiomyopathy. Semin Oncol 28 (4 Suppl 12):2-7

4. Shepard HM, Lewis GD, Sarup JC, Fendly BM, Maneval D, Mordenti J, Figari I, Kotts CE, Palladino MA, Jr., Ullrich A, et al. (1991) Monoclonal antibody therapy of human cancer: taking the HER2 protooncogene to the clinic. J Clin Immunol 11 (3):117-127

5. Slamon DJ, Leyland-Jones B, Shak S, Fuchs H, Paton V, Bajamonde A, Fleming T, Eiermann W, Wolter J, Pegram M, Baselga J, Norton L (2001) Use of chemotherapy plus a monoclonal antibody against HER2 for metastatic breast cancer that overexpresses HER2. N Engl J Med 344 (11):783-792. doi:10.1056/NEJM200103153441101

6. Piccart-Gebhart MJ, Procter M, Leyland-Jones B, Goldhirsch A, Untch M, Smith I, Gianni L, Baselga J, Bell R, Jackisch C, Cameron D, Dowsett M, Barrios CH, Steger G, Huang CS, Andersson M, Inbar M, Lichinitser M, Lang I, Nitz U, Iwata H, Thomssen C, Lohrisch C, Suter TM, Ruschoff J, Suto T, Greatorex V, Ward C, Straehle C, McFadden E, Dolci MS, Gelber RD, Herceptin Adjuvant Trial Study T (2005) Trastuzumab after adjuvant chemotherapy in HER2positive breast cancer. N Engl J Med 353 (16):1659-1672. doi:10.1056/NEJMoa052306

7. Romond EH, Perez EA, Bryant J, Suman VJ, Geyer CE, Jr., Davidson NE, Tan-Chiu E, Martino S, Paik S, Kaufman PA, Swain SM, Pisansky TM, Fehrenbacher L, Kutteh LA, Vogel VG, Visscher DW, Yothers G, Jenkins RB, Brown AM, Dakhil SR, Mamounas EP, Lingle WL, Klein PM, Ingle JN, Wolmark N (2005) Trastuzumab plus adjuvant chemotherapy for operable HER2positive breast cancer. N Engl J Med 353 (16):1673-1684. doi:10.1056/NEJMoa052122

8. Dawood S, Broglio K, Buzdar AU, Hortobagyi GN, Giordano SH (2010) Prognosis of women with metastatic breast cancer by HER2 status and trastuzumab treatment: an institutional-based review. J Clin Oncol 28 (1):92-98. doi:10.1200/JCO.2008.19.9844

9. Klein PM, Dybdal N (2003) Trastuzumab and cardiac dysfunction: update on preclinical studies. Semin Oncol 30 (5 Suppl 16):49-53

10. Seidman A, Hudis C, Pierri MK, Shak S, Paton V, Ashby M, Murphy M, Stewart SJ, Keefe D (2002) Cardiac dysfunction in the trastuzumab clinical trials experience. J Clin Oncol 20 (5):12151221

11. Perez EA, Rodeheffer R (2004) Clinical cardiac tolerability of trastuzumab. J Clin Oncol 22 (2):322-329. doi:10.1200/JCO.2004.01.120

12. Jahanzeb M (2008) Adjuvant trastuzumab therapy for HER2-positive breast cancer. Clin Breast Cancer 8 (4):324-333. doi:10.3816/CBC.2008.n.037

13. Fedele C, Riccio G, Coppola C, Barbieri A, Monti MG, Arra C, Tocchetti CG, D'Alessio G, Maurea N, De Lorenzo C (2012) Comparison of preclinical cardiotoxic effects of different ErbB2 inhibitors. Breast Cancer Res Treat 133 (2):511-521. doi:10.1007/s10549-011-1783-9

14. Jassal DS, Han SY, Hans C, Sharma A, Fang T, Ahmadie R, Lytwyn M, Walker JR, Bhalla RS, Czarnecki A, Moussa T, Singal PK (2009) Utility of tissue Doppler and strain rate imaging in the early detection of trastuzumab and anthracycline mediated cardiomyopathy. $\mathrm{J}$ Am Soc Echocardiogr 22 (4):418-424. doi:10.1016/j.echo.2009.01.016

15. Riccio G, Esposito G, Leoncini E, Contu R, Condorelli G, Chiariello M, Laccetti P, Hrelia S, D'Alessio G, De Lorenzo C (2009) Cardiotoxic effects, or lack thereof, of anti-ErbB2 immunoagents. FASEB J 23 (9):3171-3178. doi:10.1096/fj.09-131383

16. Gordon LI, Burke MA, Singh AT, Prachand S, Lieberman ED, Sun L, Naik TJ, Prasad SV, Ardehali H (2009) Blockade of the erbB2 receptor induces cardiomyocyte death through mitochondrial and reactive oxygen species-dependent pathways. Journal of Biological Chemistry 284 (4):2080-2087. doi:10.1074/jbc.M804570200 
17. Rochette L, Guenancia C, Gudjoncik A, Hachet O, Zeller M, Cottin Y, Vergely C (2015) Anthracyclines/trastuzumab: new aspects of cardiotoxicity and molecular mechanisms. Trends Pharmacol Sci 36 (6):326-348. doi:10.1016/j.tips.2015.03.005

18. De Pergola G, Silvestris F (2013) Obesity as a major risk factor for cancer. J Obes 2013:291546. doi:10.1155/2013/291546

19. Zeller M, Steg PG, Ravisy J, Lorgis L, Laurent Y, Sicard P, Janin-Manificat L, Beer JC, Makki H, Lagrost AC, Rochette L, Cottin Y, Group RSW (2008) Relation between body mass index, waist circumference, and death after acute myocardial infarction. Circulation 118 (5):482-490. doi:10.1161/CIRCULATIONAHA.107.753483

20. Rodvold KA, Rushing DA, Tewksbury DA (1988) Doxorubicin clearance in the obese. J Clin Oncol 6 (8):1321-1327

21. Mitra MS, Donthamsetty S, White B, Mehendale HM (2008) High fat diet-fed obese rats are highly sensitive to doxorubicin-induced cardiotoxicity. Toxicol Appl Pharmacol 231 (3):413-422. doi:10.1016/j.taap.2008.05.006

22. de Azambuja E, McCaskill-Stevens W, Francis P, Quinaux E, Crown JP, Vicente M, Giuliani R, Nordenskjold B, Gutierez J, Andersson M, Vila MM, Jakesz R, Demol J, Dewar J, Santoro A, Lluch A, Olsen S, Gelber RD, Di Leo A, Piccart-Gebhart M (2010) The effect of body mass index on overall and disease-free survival in node-positive breast cancer patients treated with docetaxel and doxorubicin-containing adjuvant chemotherapy: the experience of the BIG 02-98 trial. Breast Cancer Res Treat 119 (1):145-153. doi:10.1007/s10549-009-0512-0

23. Ryu SY, Kim CB, Nam CM, Park JK, Kim KS, Park J, Yoo SY, Cho KS (2001) Is body mass index the prognostic factor in breast cancer?: a meta-analysis. J Korean Med Sci 16 (5):610-614

24. Habbout A, Li N, Rochette L, Vergely C (2013) Postnatal overfeeding in rodents by litter size reduction induces major short- and long-term pathophysiological consequences. J Nutr 143 (5):553562. doi:10.3945/jn.112.172825

25. Habbout A, Guenancia C, Lorin J, Rigal E, Fassot C, Rochette L, Vergely C (2013) Postnatal overfeeding causes early shifts in gene expression in the heart and long-term alterations in cardiometabolic and oxidative parameters. PLoS One 8 (2):e56981. doi:10.1371/journal.pone.0056981

26. Habbout A, Delemasure S, Goirand F, Guilland JC, Chabod F, Sediki M, Rochette L, Vergely C (2012) Postnatal overfeeding in rats leads to moderate overweight and to cardiometabolic and oxidative alterations in adulthood. Biochimie 94 (1):117-124. doi:10.1016/j.biochi.2011.09.023

27. Walker JR, Sharma A, Lytwyn M, Bohonis S, Thliveris J, Singal PK, Jassal DS (2011) The cardioprotective role of probucol against anthracycline and trastuzumab-mediated cardiotoxicity. J Am Soc Echocardiogr 24 (6):699-705. doi:10.1016/j.echo.2011.01.018

28. Milano G, Raucci A, Scopece A, Daniele R, Guerrini U, Sironi L, Cardinale D, Capogrossi MC, Pompilio G (2014) Doxorubicin and trastuzumab regimen induces biventricular failure in mice. J Am Soc Echocardiogr 27 (5):568-579. doi:10.1016/j.echo.2014.01.014

29. Guenancia C, Li N, Hachet O, Rigal E, Cottin Y, Dutartre P, Rochette L, Vergely C (2015) Paradoxically, iron overload does not potentiate doxorubicin-induced cardiotoxicity in vitro in cardiomyocytes and in vivo in mice. Toxicol Appl Pharmacol 284 (2):152-162. doi:10.1016/j.taap.2015.02.015

30. Parekh N, Chandran U, Bandera EV (2012) Obesity in cancer survival. Annu Rev Nutr 32:311342. doi:10.1146/annurev-nutr-071811-150713

31. Zeglinski M, Ludke A, Jassal DS, Singal PK (2011) Trastuzumab-induced cardiac dysfunction: A 'dual-hit'. Exp Clin Cardiol 16 (3):70-74 


\section{Tables}

Table 1. Forward and reverse sequences of primers used for the amplification of mouse genes

\begin{tabular}{ccc}
\hline \hline $\boldsymbol{H P R T}$ & Forward (5' to 3') & Reverse (5' to 3') \\
\hline $\boldsymbol{A N P}$ & CTGGTGAAAAGGACCTCTCG & TGAAGTACTCATTATAGTCAAGGGCA \\
$\boldsymbol{B N P}$ & GAGAAGATGCCGGTAGAAGA & AAGCACTGCCGTCTCTCAGA \\
$\boldsymbol{\beta} \boldsymbol{M} \boldsymbol{M H C}$ & GCCAACACCAACCTGTCCAAGTTC & GTGCTGCCTTGAGACCGAA \\
& & \\
\hline
\end{tabular}


Table 2. Ratio $\left(\mathrm{x} 10^{3}\right)$ of left ventricular (LV) weight, measured by echocardiography, and body weight

\begin{tabular}{|c|c|c|c|c|c|c|}
\hline $\begin{array}{c}\text { LV weight/ } \\
\text { body weight } \\
\left(\mathrm{x} 10^{3}\right)\end{array}$ & D0 & $\mathrm{D} 10$ & $\mathrm{D} 20$ & $\mathrm{D} 0$ & $\mathrm{D} 10$ & D20 \\
\cline { 2 - 7 } & $4.34 \pm 0.13$ & $4.26 \pm 0.21$ & $4.22 \pm 0.23$ & $4.00 \pm 0.09^{*}$ & $3.96 \pm 0.10^{*}$ & $3.99 \pm 0.09^{*}$ \\
\hline Control & $3.99 \pm 0.15$ & $3.74 \pm 0.15$ & $3.78 \pm 0.18$ & $3.73 \pm 0.13$ & $3.99 \pm 0.19$ & $3.88 \pm 0.14$ \\
\hline DOX & $4.11 \pm 0.22$ & $4.10 \pm 0.28$ & $4.22 \pm 0.25$ & $3.85 \pm 0.10$ & $4.22 \pm 0.13$ & $3.95 \pm 0.15$ \\
\hline TRZ & $4.02 \pm 0.08$ & $4.04 \pm 0.12$ & $4.30 \pm 0.08^{\dagger}$ & $4.17 \pm 0.10$ & $4.23 \pm 0.09$ & $4.38 \pm 0.09^{\dagger}$ \\
\hline DOX + TRZ & & & & & & \\
\hline
\end{tabular}

*; significantly different from NL group, $\mathrm{p}<0.05$

$\dagger$; significantly different from DO and D10, $\mathrm{p}<0.05$ 
Table 3. Left ventricular parameters

\begin{tabular}{|c|c|c|c|}
\hline Variables & Baseline (Day 0) & Day 10 & Day 20 \\
\hline \multicolumn{4}{|l|}{ HR (beats/min) } \\
\hline NL CONT & $392 \pm 51$ & $398 \pm 40$ & $409 \pm 38$ \\
\hline NL DOX & $390 \pm 47$ & $388 \pm 51$ & $407 \pm 56$ \\
\hline NL TRZ & $356 \pm 24$ & $376 \pm 25$ & $388 \pm 27$ \\
\hline NL DOX-TRZ & $354 \pm 56$ & $370 \pm 42$ & $373 \pm 40$ \\
\hline SL CONT & $388 \pm 14$ & $391 \pm 27$ & $432 \pm 116$ \\
\hline SL DOX & $402 \pm 49$ & $395 \pm 56$ & $398 \pm 57$ \\
\hline SL TRZ & $393 \pm 45$ & $345 \pm 23$ & $400 \pm 72$ \\
\hline SL DOX-TRZ & $356 \pm 39$ & $362 \pm 40$ & $375 \pm 22$ \\
\hline \multicolumn{4}{|l|}{$\operatorname{LVEF}(\%)$} \\
\hline NL CONT & $57 \pm 5$ & $57 \pm 4$ & $56 \pm 4$ \\
\hline NL DOX & $60 \pm 4$ & $57 \pm 4$ & $58 \pm 3$ \\
\hline NL TRZ & $58 \pm 5$ & $54 \pm 7$ & $52 \pm 2$ \\
\hline NL DOX-TRZ & $59 \pm 2$ & $55 \pm 7$ & $52 \pm 6 \dagger$ \\
\hline SL CONT & $56 \pm 3$ & $56 \pm 3$ & $55 \pm 3$ \\
\hline SL DOX & $61 \pm 5$ & $49 \pm 7+*$ & $47 \pm 4 \uparrow *$ \\
\hline SL TRZ & $58 \pm 4$ & $53 \pm 3$ & $52 \pm 5$ \\
\hline SL DOX-TRZ & $58 \pm 3$ & $49 \pm 4 v^{*}$ & $48 \pm 5 \dagger$ \\
\hline \multicolumn{4}{|l|}{$\operatorname{LVEDD}(\mathrm{mm})$} \\
\hline NL CONT & $4.2 \pm 0.3$ & $4.2 \pm 0.2$ & $4.3 \pm 0.3$ \\
\hline NL DOX & $4.0 \pm 0.2$ & $4.0 \pm 0.2$ & $4.0 \pm 0.3$ \\
\hline NL TRZ & $4.1 \pm 0.4$ & $4.1 \pm 0.3$ & $4.1 \pm 0.2$ \\
\hline NL DOX-TRZ & $4.0 \pm 0.2$ & $4.0 \pm 0.2$ & $4.0 \pm 0.1$ \\
\hline SL CONT & $4.3 \pm 0.2$ & $4.2 \pm 0.2$ & $4.2 \pm 0.2$ \\
\hline SL DOX & $4.1 \pm 0.3$ & $4.4 \pm 0.3 \dagger *$ & $4.3 \pm 0.2 \uparrow *$ \\
\hline SL TRZ & $4.0 \pm 0.2$ & $4.3 \pm 0.3$ & $4.1 \pm 0.2$ \\
\hline SL DOX-TRZ & $4.2 \pm 0.3$ & $4.4 \pm 0.3^{*}$ & $4.4 \pm 0.2 *$ \\
\hline \multicolumn{4}{|l|}{ LVMN (mg/g) } \\
\hline NL CONT & $4.3 \pm 0.5$ & $4.3 \pm 0.8$ & $4.2 \pm 0.9$ \\
\hline NL DOX & $3.9 \pm 0.7$ & $3.8 \pm 0.7$ & $3.8 \pm 0.8$ \\
\hline NL TRZ & $4.1 \pm 0.5$ & $4.1 \pm 0.6$ & $4.2 \pm 0.6$ \\
\hline NL DOX-TRZ & $4.0 \pm 0.3$ & $4.0 \pm 0.4$ & $4.3 \pm 0.3$ \\
\hline SL CONT & $4.0 \pm 0.3$ & $3.9 \pm 0.3$ & $4.0 \pm 0.3$ \\
\hline SL DOX & $3.7 \pm 0.7$ & $4.0 \pm 0.9$ & $3.9 \pm 0.7$ \\
\hline SL TRZ & $3.9 \pm 0.3$ & $4.2 \pm 0.3$ & $4.0 \pm 0.4$ \\
\hline SL DOX-TRZ & $4.2 \pm 0.4$ & $4.5 \pm 0.8$ & $4.4 \pm 0.3$ \\
\hline \multicolumn{4}{|l|}{$\mathrm{E} / \mathrm{A}$ ratio } \\
\hline NL CONT & $1.4 \pm 0.1$ & $1.4 \pm 0.2$ & $1.4 \pm 0.2$ \\
\hline NL DOX & $1.4 \pm 0.1$ & $1.5 \pm 0.2$ & $1.3 \pm 0.2$ \\
\hline NL TRZ & $1.5 \pm 0.1$ & $1.4 \pm 0.1$ & $1.3 \pm 0.1$ \\
\hline NL DOX-TRZ & $1.6 \pm 0.2$ & $1.6 \pm 0.6$ & $1.5 \pm 0.5$ \\
\hline SL CONT & $1.4 \pm 0.3$ & $1.4 \pm 0.2$ & $1.5 \pm 0.6$ \\
\hline SL DOX & $1.5 \pm 0.2$ & $1.4 \pm 0.2$ & $1.4 \pm 0.2$ \\
\hline SL TRZ & $1.5 \pm 0.2$ & $1.4 \pm 0.1$ & $1.5 \pm 0.2$ \\
\hline SL DOX-TRZ & $1.5 \pm 0.2$ & $1.4 \pm 0.1$ & $1.3 \pm 0.1$ \\
\hline
\end{tabular}

Data are means \pm standard deviation. DOX indicates doxorubicin; HR: heart rate; LVEDD: left ventricular end-diastolic diameter; LVEF: left ventricular ejection fraction; LVMN: left ventricular mass normalized to body weight; $n$ : number of mice; NL: normal litter; SL: small litter. TRZ: trastuzumab. $\dagger p<0.01$ vs DO in each group, * $p<0.01$ compared with NL group with the same treatment, using 2-way ANOVA for repeated measures with a Tukey test as the post-hoc analysis 
Figure Legends

Figure 1. Study protocol for the assessment of the cardiac effects of DOX, TRZ or their combination in 4-month-old control mice from a normal litter (NL) and overweight mice from a small litter (SL): Eight study groups were created, according to the single intra-peritoneal (IP) injection of DOX (DOX, 6mg/kg), TRZ (TRZ, 10mg/kg) or DOX+TRZ (DOX-TRZ) or saline at D1. At D0, D10 and D20 all mice underwent transthoracic echocardiography. A subset of mice was killed 48 hours after the injections (D3) and blood and tissue samples were harvested. At the end of the protocol, all mice were killed and blood and tissue samples were harvested.

Figure 2. Evolution of body weight in control mice from a normal litter (NL) and overweight mice from a small litter (SL) after a single intra-peritoneal (IP) injection at D1 of (A) saline, (B) DOX (DOX, 6mg/kg), (C) TRZ (TRZ, 10mg/kg) or (D) DOX+TRZ (DOX-TRZ).

* Different from NL group, $\mathrm{p}<0.01$

$\dagger$ Different from D0, $\mathrm{p}<0.05$

Figure 3. Evolution of left ventricular ejection fraction (LVEF) in control mice from a normal litter (NL) and overweight mice from a small litter (SL) after a single intra-peritoneal (IP) injection at D1 of (A) saline, (B) DOX (DOX, 6mg/kg), (C) TRZ (TRZ, 10mg/kg) or (D) DOX+TRZ (DOX-TRZ).

* Different from NL group, $\mathrm{p}<0.01$

$\dagger$ Different from D0, $\mathrm{p}<0.01$

Figure 4. Relative mRNA expression of (A) brain natriuretic peptide (BNP), (B) atrial natriuretic peptide (ANP) and $(C)$ beta-myosin heavy chain $(\beta-M H C)$ at $\mathrm{D3}$ in control mice from a normal litter (NL) and overweight mice from a small litter (SL) after single intraperitoneal (IP) injection of DOX (DOX, 6mg/kg), TRZ (TRZ, 10mg/kg) or DOX+TRZ (DOXTRZ) or saline at D1. 